\title{
Clinical, pathological and molecular predictors of Fulvestrant long-term benefit in Hormone Receptor- positive / HER2 negative advanced breast cancer
}

Rosalba Torrisi ( $\nabla$ rosalba.torrisi@humanitas.it)

IRCCS Humanitas Research Hospital

\section{Valentina Vaira}

Fondazione IRCCS Ca' Granda Ospedale Maggiore Policlinico

\section{Laura Giordano}

IRCCS Humanitas Research Hospital

\section{Annarita Destro}

IRCCS Humanitas Research Hospital

\section{Vera Basilico}

Humanitas Mater Domini

\section{Saveria Mazzara}

European Institute of Oncology

\section{Piermario Salvini}

Humanitas Gavazzeni

\section{Gabriella Gaudioso}

Fondazione IRCCS Ca' Granda Ospedale Maggiore Policlinico

\section{Bethania Fernandes}

IRCCS Humanitas Research Hospital

Noemi Rudini

IRCCS Humanitas Research Hospital

Giovanna Masci

IRCCS Humanitas Research Hospital

\section{Armando Santoro}

Humanitas University

\section{Research Article}

Keywords: hormone receptor positive advanced breast cancer, long-term benefit, Fulvestrant, PI3KCA mutations, miRNAs

Posted Date: January 31st, 2022 
DOI: https://doi.org/10.21203/rs.3.rs-1300009/v1

License: (c) (1) This work is licensed under a Creative Commons Attribution 4.0 International License. Read Full License 


\section{Abstract}

Purpose: The identification of factors predicting the benefit of treatments is a major goal for medical oncologists.

Methods We retrospectively investigated in metastatic tumor tissues of women treated with fulvestrant for HR+/HER2 negative advanced breast cancer clinical, pathological and molecular features associated with long-term benefit from treatment defined as being progression-free at 18 months. Specifically, we analyzed ESR1 and PI3KCA mutations and miRNA profiles.

Results: Fifty-nine patients were evaluable (median age $67 \mathrm{yrs}$, range 32-92). Overall mPFS was 10.7 months (range 2.2-14) while 18-month PFS rate was $27 \%$; only the lack of visceral metastases significantly predicted the likelihood of being progression-free at 18 months $(\mathrm{OR}=0.2595 \% \mathrm{Cl}$ 0.07-0.9 $\mathrm{p}=.035)$. PI3KCA mutations were found in $36 \%$ of patients and were associated with a numerically shorter mPFS but not with a lower likelihood of being progression-free at 18 months.

As of miRNAs, miR-549a, miR-644a, miR-16-5p were negatively associated with 18-month PFS while let$7 c-5 p$ was positively associated with outcome. In addition, $\operatorname{miR}-520 d-3 p$ and $m i R-548 g-3 p$ values were significantly lower while miR-603, miR-181a and miR-199a-miR-199b-3p values were significantly higher in patients achieving 18-month PFS. In silico analysis of predicted targets suggested that miR-520d-3p and miR-548g-3p decrease might induce Hippo and Wnt signaling, while high levels of miR-603, mir-181a$5 p$, miR-199a-miR-199b-3p might repress endocrine resistance thus potentially promoting the response to treatment.

Conclusions: Our clinical findings are consistent with literature data, while the mutation and miRNA results provide some clues on the molecular mechanisms involved in fulvestrant activity and resistance to be confirmed in larger cohorts of breast cancer patients.

\section{Introduction}

About two thirds of breast cancers are hormone receptor-positive HER2 negative (HR+/HER2-) (1). The combination of endocrine therapy (ET) and CDK4/6 inhibitors (CDK4/6i) has established as standard treatment for HR+/HER2- advanced breast cancer (ABC) (2). Four randomized trials including thousands of patients have shown an impressive and consistent advantage of 10-14 months in progression free survival (PFS) for the association of any of the three CDK 4/6i investigated with non-steroidal aromatase inhibitors (NSAI) in endocrine sensitive $A B C$ as compared to the endocrine agent alone (3).

On the other hand, since multiple mechanisms of resistance underlying CDK4/6i failure have been proposed, no definite treatment indications after progression to $C D K 4 / 6 \mathrm{i}$ exist and international guidelines indicate that a further endocrine manipulation as well as a switch to chemotherapy may be offered $(2,4)$. 
The Selective Estrogen Receptor Downregulator (SERD) fulvestrant has established as a valuable option in the treatment of advanced HR+/HER2- ABC. Head-to-head comparison with NSAl in the $1^{\text {st }}$ line treatment has shown the superiority of fulvestrant (5) and in the CONFIRM study, NSAI-resistant patients achieving a clinical benefit with fulvestrant had a prolonged disease control (> 16 months) (6).

Thus it appears crucial to identify clinical and pathological factors which may predict the subset of patients who maintain endocrine sensitiveness and may be spared more aggressive treatments for a clinically relevant span of time and, on the other hand, patients who are likely to derive no or minimal benefit from fulvestrant who deserve more effective treatments upfront. At the present time no predictors of response or resistance have been established.

MicroRNAs (miRNAs) are a class of small, non-coding RNA that regulate the gene expression of target mRNAs at post-transcriptional level $(7,8)$. MiRNAs have been implicated in regulating breast cancer cell proliferation, cell death, apoptosis, immune response, cell cycle energetics, senescence, invasion, and metastasis (9). Moreover, miRNAs are emerging as novel potential predictive/prognostic biomarkers of disease and response to therapies $(8,9)$. Several miRNAs have been found deregulated in breast cancer cell lines (BCCL), on cancer specimens and in serum of breast cancer patients and have been correlated with prognostic features and with response and resistance to endocrine manipulations (9).

In the present study we investigated in a series of women with HR+/HER2- ABC treated with fulvestrant whether a panel of miRNAs was associated with long-term benefit from fulvestrant. According to previous literature data long-term benefit was defined as treatment duration without progression lasting at least 18 months (10).

In addition, since endocrine resistance has been associated with mutations of ESR1 and PI3KCA the occurrence of these mutations was assessed and correlated with miRNA profiles and with outcome $(11,12)$.

\section{Patients And Methods}

This observational retrospective multicentric study aimed to describe the clinical outcome of patients with $\mathrm{HR}+/ \mathrm{HER} 2-\mathrm{ABC}$ who received fulvestrant as part of their routine treatment at 3 Italian Institutions and to correlate it with clinical, pathological and molecular features.

PATIENTS: Eligible patients were pre/peri- and postmenopausal women aged $\geq 18$ years diagnosed with $\mathrm{HR}+$ (defined as ER and/or PgR $\geq 10 \%$ ) and HER2- (defined as IHC 0, 1+ or 2+ with FISH negative) inoperable locally advanced and/or metastatic breast cancer for whom tissue specimen of advanced or metastatic disease obtained for diagnosis was available. Pre/perimenopausal patients received concomitant ovarian suppression with $\mathrm{GnRH}$ analogues. Fulvestrant was administered $500 \mathrm{mg}$ i.m. q28 days with loading dose after 14 days as any line of treatment. Patients treated before October 2011 received fulvestrant $250 \mathrm{mg}$ i.m q28 days. 
All tumor samples were obtained after diagnosis of metastatic disease and before fulvestrant treatment and were analyzed for routine diagnosis at the same pathologic department by breast dedicated pathologists.

Patient medical charts were reviewed and tumor response was assessed. Since this is a retrospective study response could not be evaluated according to RECIST 1.1 criteria in all patients, but CT scans and other imaging were reviewed in order to comply with RECIST criteria whenever possible. Information on age at diagnosis, menopausal status, previous adjuvant therapies, previous therapies for metastatic disease, pathological features of the metastatic tumor, sites of metastatic disease, best response, and disease progression if occurred and last follow up visit were collected.

All patients signed informed consent

This study was approved by the institutional ethical committee of the coordinating center (Humanitas Research Hospital) and of the other participating Institutions and was conducted in compliance with Helsinki Declaration.

MUTATION ANALYSES: DNA was extracted from paraffin-embedded breast cancer tissue at least $60 \%$ tumor enriched using the Maxwell RSC DNA FFPE Kit (Promega) according to the manufacturer's instructions. If required manual microdissection was performed. Both PIK3CA (E542K, E542Q, C420R, H1047P, H1047R, H1047L, E545K, E545Q, Q546K, Q546E, E545A, E545G, E545V) and ESR1 (E380Q, S463P, D538G, D538V, L536H, L536R, L536P, Y537C, Y537S, Y537N, P535H) hot spot mutations were analyzed using the Sequenom MassARRAY platform (SEQUENOM ${ }^{\circledR} \mathrm{Inc}$ ). The PI3KCA and ESR codons were amplified and processed using Diatech Pharmacogenetics reagents according to the manufacturer's instructions used for Myriapod Lung status assay. The sequences and molecular weights of extension primers and products were listed in table S1b. A cutoff of $5 \%$ of allelic frequency was generally guaranteed.

MiRNA ANALYSES Formalin-fixed paraffin embedded (FFPE) breast cancer samples underwent total RNA isolation using the using the Maxwell RSC RNA FFPE kit (Promega, Madison, WI, USA) according to the manufacturer's protocol. Quantification and RNA quality assessment were performed using a Nanodrop 2000 spectrophotometer (NanoDrop Products, Wilmington, DE, USA).

miRNA expression profiling was performed using the nCounter Human v3 miRNA Panel (NanoString Technologies, Seattle, WA, USA), which contains 798 unique miRNA barcodes. RNA was prepared according to the manufacturer's protocol in a total of 5 runs. The nCounter Flex instrument was used and all counts were gathered by scanning on HIGH mode for 555 fields of view per sample. Raw data were analyzed as described in nCounter Data Analysis Guidelines for miRNA (13). Specifically, the background threshold was set at 20; any value below this threshold was converted to zero. After that, miRNA counts were normalized to the geometric mean of the 50 miRNAs with the highest counts. Then, normalized miRNA values were subjected to statistical analyses. Nanostring data processing was carried out using R 3.6.1 software (R Core Team) (14). 
Predicted miRNA targets were searched using the miRTargetLink2 web tool, selecting only validated targets. Then, miRNAs targets were imported in STRING database and analyzed for significantly enriched GO terms (Biological process and Molecular function) and KEGG Pathways using the human whole genome as statistical background as previously described (DOI: 10.3389/fonc.2021.643280)

\section{STATISTICAL ANALYSES}

Categorical variables were expressed as number and percentages, while continuous variables as medians with the respective range.

Clinical and demographical data and miRNAs were associated with outcome as continuous value in terms progression free survival (PFS) by the cox proportional hazard model to calculate hazard ratio and their corresponding 95\% confidence intervals. Moreover, the impact of miRNAs with long-term survival was described considering as success patients with a PFS $\geq 18$ months and estimating Odds ratios with their corresponding 95\% confidence intervals with a logistic regression model. Distribution of miRNAs between patients achieving or not 18-month PFS were estimated by Wilcoxon t test. For patients presenting miRNA assessment both at baseline and at progression, a normalized variation was calculated as (miRNAto - miRNApd)/miRNAto to explore a possible miRNA profile association with progression.

Considering the small sample size all evaluation were expolorative in nature. A statistical p value of 0.05 was arbitrary set to present results to be better investigate in subsequent studies.

All analyses were performed using SAS version 9.4 (SAS Institute Inc, Cary, NC).

\section{Results}

From a database of 202 women with HR+/HER2- ABC who received treatment with fulvestrant from 2005 to 2017 at the 3 participating Institutions were retrieved 74 patients who underwent a diagnostic biopsy for metastatic disease. Fourteen patients were excluded because there was no residual tissue for further analyses and one additional patient did not have clinical information. Fifty-nine patients were evaluable for the molecular analyses. Tumor tissue was obtained from pleura (16), breast, when biopsy of synchronous metastatic sites was not feasible, (15), lymph nodes (9), bone (7), skin (5), liver (4), other sites (3). Seven patients had also a biopsy obtained after progression on fulvestrant.

Baseline patient characteristics are summarized in Table 1. Median age was 67 yrs (range 32-92).

Clinical benefit was obtained in 36 patients (61\%). No clinical variable (tumor subtype, number of metastatic sites, number of previous lines, disease status at fulvestrant) was statistically significantly associated with median PFS ( $\mathrm{mPFS}$ ), although a trend towards a shorter mPFS in patients with visceral metastases vs patients with no visceral involvement was observed (7.5 months vs 12.6, respectively $p=0.059)$. At the same time, patients with both HR positive tumors had a not statistically significant 
longer PFS as compared with patients with PgR negative tumors (13.8 vs 7,5 months, respectively, $p=$ .099)

At 18 months 16 patients were progression free and alive with an 18-month PFS rate of $27 \%$. Analyses of clinical and pathological variables showed that only the lack of visceral metastases significantly predicted the likelihood of being progression-free at 18 months $(O R=0.2595 \% \mathrm{Cl} 0.07-0.9 p=.035)$.

Mutation analyses ESR1 and PI3KCA mutations were assessed in 53 patients. ESR1 mutations were observed in the pretreatment biopsy in 5 patients, all previously treated with NSAI: D538V and Y537S (2 patients) and E380Q (1 patient). In the 7 patients with repeated biopsies ESR1 mutations were observed in both biopsies in 1 patient (Y537S) while they were detected only in the post-progression biopsy in 2 cases (Y537S and D538G). Since ESR1 mutations were found only in $9 \%$ of the patients, no correlation with other features was investigated

PI3KCA mutations were found in pretreatment biopsies in 19 (36\%) patients: H1047R (10 patients), E542K (4 patients), E545K and H1047L (2 patients), Q546K (1 patient). Among the 7 patients with repeated biopsies only 2 patients had PI3KCA mutations in both samples while 1 patient lost the PI3KCA mutation and developed an ESR1 mutation in the post-progression biopsy.

PFS in patients with PI3KCA mutated tumors was numerically shorter than in patients not harboring mutations (mPFS 7.6 vs 11 months, respectively, $p=0.28$ ) On the other hand the probability of being progression-free at 18 months was not different in the 2 groups ( $O R=1.0195 \% \mathrm{Cl} 0.28-3.6 p=0.99)$

miRNA analyses miRNA profiles were assessed in 50 patients since patients with bone biopsies were excluded for this analysis because of poor RNA quality.

45 miRNAs were detected in at least 30 samples, 83 miRNAs were detected in at least 20 samples and 118 miRNAs were detected in at least 10 samples.

We investigated correlation with outcome only for miRNAs which were detected in at least $20 \%(n=10)$ of samples ( detailed in supplementary table 2).

miRNAs which were significantly associated with PFS are reported in Table 2. Fourteen miRNAs were negatively associated with outcome while 2 miRNAs belonging to the let-7 family were positively associated with improved outcome.

When we considered the likelihood of being progression free at 18 months as endpoint only miR-549a, miR $-644 a$ confirmed to be negatively associated with outcome in addition to miR-16-5p, while let-7c$5 p$ was associated also with a greater likelihood of being progression free at 18 months (Table 2).

When we analyzed miRNA distribution comparing patients with or without a PFS $\geq 18$ months we found that miR-549a, miR-644a, miR-16-5p values were significantly higher while let-7c-5p value was lower in 
patients not achieving 18-month PFS, respectively. In addition to these, $\operatorname{miR}-520 d-3 p$ and $\operatorname{miR}-548 g-3 p$ were significantly and negatively associated with 18-month PFS while miR-603, mir-181a-5p and miR199a-miR-199b-3p values were statistically significantly higher in the long-term benefit cohort; moreover miR-125b-5p showed a not significant trend to be higher in patients being progression free at 18 months.

In silico analysis of predicted targets showed that cell metabolism was the main biological process involved in fulvestrant treatment. Analysis of predicted pathways suggested that miR-520d-3p and miR$548 g-3 p$ decrease might induce Hippo and Wnt signaling. On the contrary, high levels of miR-603, mir181a-5p, miR-199a-miR-199b-3p might repress endocrine resistance thus potentially promoting the antitumor activity of Fulvestrant .

Finally, no specific miRNA expression was associated with PI3KCA mutation status (data not shown). Similarly, we did not find any specific baseline miRNA profile or change at disease progression in the 7 patients for whom both a baseline and post progression biopsy were available (data not shown).

\section{Discussion}

The identification of clinical, pathological and molecular features predicting the benefit of treatments is a major goal for medical oncologists.

Fulvestrant has been proven a valuable option for treatment of HR+ABC. First-line fulvestrant was associated with a mPFS of 16.6 months which extended to 22 months in patients with bone-only metastases in a population of mostly treatment-naïve patients (5). In the cohort of patients treated as $1^{\text {st }}$ line in the MonaLEEsa-3 study mPFS in the Fulvestrant / placebo arm was 19 months, while in the control arms of $2^{\text {nd }} / 3^{\text {rd }}$ line studies with CDK $4 / 6 i$ and Fulvestrant, mPFS decreased to 9 and 4.6 months, respectively (15-17). Similar data have been reported in patients treated with Fulvestrant in real-world series (18-20).

Identification of pathological and molecular features predicting benefit from treatment in tumor samples of patients treated with fulvestrant has been attempted previously $(21,22)$. The TransCONFIRM, a translational analysis within the CONFIRM trial aimed to identify clinical-pathological features and molecular signatures in the primary tumors predicting response to fulvestrant, showed in the 112 samples analyzed that only PgR and HER2 expression and a signature of 37 genes were independently associated with PFS, (21). Of note, no correlation with ESR1 mutations on primary tumors was found (22). Christensen et al. investigated in mRNA extracted from 226 tumor samples of patients treated with fulvestrant the predictive value of a mathematical algorithm based on the expression of multiple genes (DRP) (22). The DRP was associated although not significantly with outcome in patients treated in earlier lines and unexposed to previous adjuvant endocrine therapies (23).

Our clinical data are comparable with those mentioned above, showing a mPFS of 10.6 months in a heavily pretreated cohort, since $46 \%$ of our patients were treated in $3^{\text {rd }}$ or later lines. Only the occurrence 
of visceral metastases was associated with a worse outcome, although we cannot exclude that the correlations between HR and PI3KCA mutation status and outcome were not significant only because of the limited sample size. Similarly, only the absence of visceral metastases was significantly associated with a 4-fold higher likelihood of long-term benefit from fulvestrant,

Notably, patients starting Fulvestrant after progressing to other therapies experienced a similar benefit as patients receiving treatment as maintenance after chemotherapy (mPFS=9.9 months vs 10.8 months, respectively).

The role of PI3KCA mutations in the mechanisms of resistance to fulvestrant is not clear. Results of the fulvestrant/placebo arms in studies with PI3K inhibitors are inconsistent (23). While in the BELLE-2 study mPFS in patients with PI3KCA wild-type tumors doubled that of patients with PI3KCA mutant tumors, no difference was observed in the same comparisons within the BELLE-3 and SOLAR-1 studies (23). In a recent analysis on ctDNA of patients included in the PALOMA 3 trial PI3KCA mutations while being among the most common mutations at baseline did not increase after progression and did not correlate with PFS (24).

In our study PI3KCA mutations were found in about $36 \%$ of patients, consistently with what expected in Luminal breast cancers (25) and were associated with a numerically shorter PFS but not with a lower likelihood of being progression-free at 18 months. These findings are only partially consistent with the analysis of the long-term benefit in the fulvestrant/placebo arm of the PALOMA 3 trial since in this study only $6 \%$ of patients harboring PI3KCA mutations vs $39 \%$ of patients with PI3KCA wild type tumors were treated for $\geq 18$ months (10).

Similarly to what observed in the PALOMA 3 study, the lack of PgR expression was associated with a lower likelihood of long-term benefit from Fulvestrant (10).

The rate of ESR1 mutations was lower than expected, despite more than $50 \%$ of patients had previously received a NSAI Notably ESR1 mutations were observed only in post-progression biopsy in 2 patients, one of whom had lost at the same time PI3KCA mutation, suggesting that a different mechanism of resistance to fulvestrant was developed.

A huge number of miRNAs have been associated with breast cancer with alternative and not always consistent suppressive or oncogenic properties for each miRNA and neither meta-analyses have been able to define an unequivocal expression and ro le even for more frequently expressed miRNAs $(9,26-29)$. Discrepancies among studies may be attributed to several factors as differences in patient populations, biological samples (fresh tissues, paraffin-embedded tissues and blood) and methodological procedures (qRT-PCR, NGS, microarray) but may also be related to different functions of each miRNA according to the tumor microenvironment $(9,26-29)$.

In our study we identified 9 miRNAs which were significantly and differently associated with 18-month PFS. Literature data provide evidence about 3 of these miRNAs (let-7c, miR-520d-3p, miR-181a) to be 
involved in $\mathrm{HR}+\mathrm{ABC}$ (30-35). In particular miR-520d-3p was among miRNAs which were downregulated upon estradiol stimulation in BCCL (30). On the other hand, miR-520d-3p has also been proposed to suppress ESR1 expression and to be involved in endocrine resistance $(31,32)$.

A larger amount of consistent evidence is available on the let-7 miRNA family which was demonstrated to target ER-a and negatively affect its function in ER-positive BCCL (33). In particular, let-7c targets ESR1 and is less expressed in metastatic tissue than in primary tumor and normal tissue (32). Upon analysis of clinical data from The Cancer Genome Atlas, it was suggested that low expression of let-7c in addition to other miRNAs (miR-99a and miR-125b) was associated with worse overall survival compared with patients who had high expression of these miRNAs (34).

Our findings showing that let-7c-5p was significantly and positively correlated with a higher likelihood of being progression-free at 18 months and let-7f-5p significantly directly correlated with PFS confirmed the suppressive properties for the members of the let-7 family.

In addition, we found a not significantly higher expression of miR-125b in patients achieving 18-month PFS.

Contradictory evidence is available on miR-181a-5p, which belongs to a family of largely expressed miRNAs (29). It was upregulated and associated with poor survival in metastatic breast cancer patients particularly in those with TNBC (29), but, on the other hand, it is among the most potent miRNAs repressing cell growth and counteracting estradiol-dependent cell proliferation (30). In our study a greater expression of miR-181a was positively associated with long-term fulvestrant benefit.

Also on miR-16-5p literature data are somehow inconsistent since preclinical data support tumor suppressive properties but an increased expression in TNBC as compared to normal tissues has been found as well as either a down- or upregulation in the serum of breast cancer patients as compared to healthy controls $(33,35-37)$. In our study miR-16-5p was associated with a decreased probability of being progression-free at 18 months.

In silico analysis of the targets of miRNAs inversely associated with long-term outcome showed that oncogene-induced cell senescence was the most affected biological processes. Analysis of predicted pathways suggested that miR-520d-3p and miR-548g-3p decrease might induce Hippo and Wnt signaling. On the other hand miR- 603 and miR-181a-5p and miR-199a-miR-199b-3p were predicted to suppress endocrine resistance.

The miRNAs we found significantly associated with long-term benefit or resistance to fulvestrant were not among miRNAs differently expressed in fulvestrant-resistant cell lines as compared with parental MCF-7 cells $(38,39)$. Moreover, in our series miR-221 and mir-222 which had been associated with fulvestrant resistance in BCCL were detected only in 2 and 8 samples, respectively and therefore were not further correlated with outcome; at the same time miR-21, despite being confirmed among the most highly expressed miRNAs, did not show any association with outcome $(40,41)$. 
We acknowledge that our study has some limitations. First of all, the limited number of patients with available metastatic tissue which resulted in a small sample considering the large number of putative predictive variables examined especially for miRNA analyses; then the retrospective design which resulted in missing clinical information and which may have not allowed to meet with a strict application of RECIST 1.1 criteria for all patients. Moreover, we did not perform in vitro assays in BCCL to better elucidate the correlation between the miRNA profile associated with the in vivo response to fulvestrant and gene expression analyses to identify the related target genes involved. Finally, since the current use of fulvestrant monotherapy is recommended only after CDK 4/6i, we cannot rule out that this treatment can induce molecular patterns different from those observed in our CDK 4/6i naïve population.

\section{Conclusions}

Altogether, our clinical findings are consistent with literature data from randomized trials and real-world series, confirming the poor prognostic role of visceral metastases. Our data do not support an association between PI3KCA mutations and long-term benefit from treatment. Furthermore, we propose a new panel of miRNAs which were associated with long-term benefit of endocrine therapy and which are mainly involved in cell metabolism and endocrine resistance. Our results provide some additional clues on the mechanisms involved in fulvestrant activity and resistance although underlying molecular pathways should be further elucidated and confirmed in larger cohorts of breast cancer patients .

\section{Abbreviations}

HR Hormone receptors

ABC Advanced breast cancer

ET Endocrine Therapy

PFS Progression-free survival

NSAI Non-steroidal aromatase inhibitor

miRNA micro-RNA

BCCL Breast Cancer Cell Lines

ESR1 Estrogen receptor gene

PI3K Phosphoinositide 3-kinase

FFPE Formalin-fixed paraffin-embedded

Go Gene Ontology analysis 
KEGG Kyoto Encyclopaedia of Genes and Genomes

TNBC Triple Negative Breast Cancer

\section{Declarations}

\section{ACKNOWLEDGEMENTS}

The authors are indebted with prof. Stefano Paolo Pileri for his helpful comments in the processing of miRNA data

Funding This study was supported by an unrestricted grant from Astra Zeneca

Authors' contributions All authors contributed to the study conception and design. RT, VB, PS, GM contributed to patient enrollment and management. VV, AD, BF, NR GG performed molecular analyses. SM analyzed Nanostring data, LG performed statistical analyses. The first draft of the manuscript was written by RT and all authors revised all the versions of the manuscript. All authors read and approved the final manuscript.

Availability of data and material The datasets generated and analysed during the current study are available from the corresponding author on reasonable request.

\section{Compliance with ethical standards}

\section{Conflict of interest}

Rosalba Torrisi: Astra Zeneca, Eisai, Pfizer, Eli Lilly, Exact Sciences, MSD

Armando Santoro BMS ,Servier , Gilead, Pfizer, Eisai , Bayer, MSD, Takeda, Roche, Astra Zeneca, Pfizer, Eli Lilly, Novartis, Aqule, Sandoz, Abb-Vie

The other authors declare no conflict of interest

Ethics approval The study was approved by the Ethical Committees of all the participating Institutions .The study was conducted in compliance with Helsinki Declaration.

Consent to participateAll patients provided written informed consent for participation to the study and for the use of their anonymized clinical data for scientific purpose

\section{References}

1. Hammond ME, Hayes DF, Dowsett M, Allred DC, Hagerty KL, Badve S, Fitzgibbons PL, Francis G, Goldstein NS, Hayes M, Hicks DG, Lester S, Love R, Mangu PB, McShane L, Miller K, Osborne CK, Paik S, Perlmutter J, Rhodes A, Sasano H, Schwartz JN, Sweep FC, Taube S, Torlakovic EE, Valenstein P, 
Viale G, Visscher D, Wheeler T, Williams RB, Wittliff JL, Wolff AC American Society of Clinical Oncology/College of American Pathologists guideline recommendations for immunohistochemical testing of estrogen and progesterone receptors in breast cancer. J Clin Oncol 2010: 28: 2784-2795

2. Cardoso F , Paluch-Shimon S, Senkus E, Curigliano G, Aapro MS, André F, Barrios CH, Bergh J, Bhattacharyya GS, Biganzoli L, Boyle F, Cardoso MJ, Carey LA, Cortés J, El Saghir NS, Elzayat M, Eniu A, Fallowfield L, Francis PA, Gelmon K, Gligorov J, Haidinger R, Harbeck N, Hu X, Kaufman B, Kaur R, Kiely BE, Kim SB, Lin NU, Mertz SA, Neciosup S, Offersen BV, Ohno S, Pagani O, Prat A, Penault-Llorca F, Rugo HS, Sledge GW, Thomssen C, Vorobiof DA, Wiseman T, Xu B, Norton L, Costa A, Winer EP.5th ESO-ESMO international consensus guidelines for advanced breast cancer (ABC 5). Ann Oncol. 2020 31:1623-1649.

3. Murphy CG. The role of cd4/6 inhibitors in breast cancer. Curr Treat Option in Oncol 2019 20: 52 DOI 10.1007/s11864-019-0651-4.

4. NCCN v 1.2021 guidelines. NCCN.org.

5. Robertson JFR, Bondarenko IM, Trishkina E, Dvorkin M, Panasci L, Manikhas A, Shparyk Y, CardonaHuerta S, Cheung KL, Philco-Salas MJ, Ruiz-Borrego M, Shao Z, Noguchi S, Rowbottom J, Stuart M, Grinsted LM, Fazal M, Ellis MJ. Fulvestrant $500 \mathrm{mg}$ versus anastrozole $1 \mathrm{mg}$ for hormone receptorpositive advanced breast cancer (FALCON): an international, randomised, double-blind, phase 3 trial. Lancet. 2016;388:2997-3005.

6. Garnett SA, Martin M, Jerusalem G, Petruzelka L, Torres R, Bondarenko, IN, Khasanov R, Verhoeven D, Pedrini JL, Smirnova I, . Lichinitser MR, Pendergrass K, Lindemann JPO, and Di Leo A. Comparing duration of response and duration of clinical benefit between fulvestrant treatment groups in the CONFIRM trial: application of new methodology. Breast Cancer Res Treat. $2013 ; 138: 149-55$

7. Di Leva G, Garofalo M, Croce CM. MicroRNAs in cancer. Annu Rev Pathol. 2014;9:287-314.

8. Vaira V, Roncalli M, Carnaghi C, Faversani A, Maggioni M, Augello C, Rimassa L, Pressiani T, Spagnuolo G, Di Tommaso L, Fagiuoli S, Rota Caremoli E, Barberis M, Labianca R, Santoro A, Bosari S.MicroRNA-425-3p predicts response to sorafenib therapy in patients with hepatocellular carcinoma. Liver Int. 2015 Mar;35(3):1077-86. doi: 10.1111/liv.12636

9. Campos-Parra AD, Mitznahuatl GC, Pedroza-Torres A, Romo RV, Reyes FIP, López-Urrutia E, PérezPlasencia C. Micro-RNA as potential predictors of response to breast cancer systemic therapy: future clinical implications. Int J Mol Sci 2017, 18:1182.

10. Cristofanilli M, DeMichele A, Giorgetti C, Turner NC, Slamon DJ, Im SA, Masuda N, Verma S, Loi S, Colleoni M, Theall KP, Huang X, Liu Y, Bartlett $\mathrm{CH}$.. Predictors of prolonged benefit from palbociclib plus fulvestrant in women with endocrine-resistant hormone receptor-positive/human epidermal growth factor receptor 2. negative metastatic breast cancer inPALOMA-3. Eur J cancer 2018; 104: 21 31

11. Jeselsohn R, Buchwalter G, De Angelis C , Brown M and Schiff R.. ESR1 mutations-a mechanism for acquired endocrine resistance in breast cancer. Nat Rev Clin Oncol 2015 10:573-83. 
12. Miller TW, Rexer BN, Garrett JT and Arteaga CL. Mutations in the phosphatidylinositol 3-kinase pathway: role in tumor progression and therapeutic implications in breast cancer. Breast Cancer Res 2011; 13: 224.

13. Tabano S, Caldiroli A, Terrasi A, Colapietro P, Grassi S, Carnevali GS, Fontana L, Serati M, Vaira V, Altamura AC, Miozzo M, Buoli M. A miRNome analysis of drug-free manic psychotic bipolar patients versus healthy controls Eur Arch Psychiatry Clin Neurosci. 2020270 :893-900. doi: 10.1007/s00406019-01057-2)

14. R Core Team. R: A language and environment for statistical computing. R Foundation for Statistical Computing, Vienna, Austria. 2014. URL http://www.R-project.org/

15. Slamon, DJ, Neven P, Chia S, Fasching PA, De Laurentiis M, Im SA, Petrakova K, Bianchi GV, Esteva FJ, Martín M, Nusch A, Sonke GS, De la Cruz-Merino L, Beck JT, Pivot X, Sondhi M, Wang Y, Chakravartty A, Rodriguez-Lorenc K, Taran T, Jerusalem G.. Overall Survival with Ribociclib plus Fulvestrant in Advanced Breast Cancer N Engl J Med 2020;382:514-24. DOI:

10.1056/NEJMoa1911149

16. Sledge Jr, GW, Toi M, Neven P, Sohn J, Inoue K, Pivot X, Burdaeva O, Okera M, Masuda N, Kaufman PA, Koh H, Grischke EM, Conte P, Lu Y, Barriga S, Hurt K, Frenzel M, Johnston S, Llombart-Cussac A. The Effect of Abemaciclib Plus Fulvestrant on Overall Survival in Hormone Receptor-Positive, ERBB2-Negative Breast Cancer That Progressed on Endocrine Therapy-MONARCH 2 A Randomized Clinical Trial. Jama Oncol. 2020; 6:116-124. doi:10.1001/jamaoncol.2019.4782

17. Turner NC, Slamon DJ, Ro J, Bondarenko I, Im SA, Masuda N, Colleoni M, DeMichele A, Loi S, Verma S, Iwata H, Harbeck N, Loibl S, André F, Puyana Theall K, Huang X, Giorgetti C, Huang Bartlett C, Cristofanilli M. Overall survival with palbociclib and fulvestrant in advanced breast cancer. $\mathrm{N}$ Engl $\mathrm{J}$ Med 2018; 379:1926-1936

18. Kawaguchi H, Masuda N, Nakayama T, Aogi K, Anan K, Ito Y, Ohtani S, Sato N, Saji S, Tokunaga E, Nakamura S, Hasegawa Y, Hattori M, Fujisawa T, Morita S, Yamaguchi M, Yamashita T, Yamamoto Y, Ohno S, Toi M. , Outcomes of fulvestrant therapy amongJapanese women with advanced breast cancer:a retrospective multicenter cohort study(JBCRG-C06; Safari). Breast Cancer ResTreat.2017; 163: $545-554$.

19. Blancas I, Fontanillas M, Conde V, Lao J, Martínez E, Sotelo MJ, Jaen A, Bayo JL, Carabantes F, Illarramendi JJ, Gordon MM, Cruz J, García-Palomo A, Mendiola C, Pérez-Ruiz E, Bofill JS, BaenaCañada JM, Jáñez NM, Esquerdo G, Ruiz-Borrego M.. Efficacy of fulvestrant in the treatment of postmenopausal women with endocrine-resistant advanced breast cancer in routine clinical practice. Clin TranslOncol 2018; 20: 862-869.

20. Palumbo R, Sottotetti F, Quaquarini E, Gambaro A, Ferzi A, Tagliaferri B, Teragni C, Licata L, Serra F, Lapidari P, Bernardo A.. Patterns of treatment and outcome with 500-mg fulvestrant in postmenopausal women with hormone receptor-positive/ HER2-negative metastatic breast cancer: a real-life multicenter Italian experience. Ther Adv Med Oncol 2019, 11: 1- 13. DOI: 10.1177/ 1758835919833864. 
21. JesehIson R, Barry WT, Migliaccio I, Biagioni C, Zhao J, De Tribolet-Hardy J, Guarducci C, Bonechi M, Laing N, Winer EP, Brown M, Leo AD, Malorni L.. TransCONFIRM: Identification of a Genetic Signature of Response to Fulvestrant in Advanced Hormone Receptor-Positive Breast Cancer. Clin Cancer Res $2016 ; 22: 5755-5764$.

22. Christensen TD, Buhl ASK, Christensen IJ, Buhl IK, Balslev E, Knoop AS, Danø H, Glavicic V, Luczak A, Langkjer ST, Linnet S, Jakobsen EH, Bogovic J, Ejlertsen B, Rasmussen A, Hansen A, Knudsen S, Jensen PB, Nielsen D. Prediction of fulvestrant efficacy in patients with advanced breast cancer: retrospective-prospective evaluation of the predictive potential of a multigene expression assay. Breast Cancer 2020; 27:266-276 https://doi.org/10.1007/s12282-019-01017-7

23. Mollon LE, Anderson EJ, Dean JL, Warholak TL, Aizer A, Platt EA, Tang DH, Davis LE. A Systematic Literature Review of the Prognostic and Predictive Value of PIK3CA Mutations in HR+/HER2Metastatic Breast Cancer. Clin Breast Cancer, 2020; 20: e232-43 a 2019

24. O'Leary B, Cutts RJ, Huang X, Hrebien S, Liu Y, André F, Loibl S, Loi S, Garcia-Murillas I, Cristofanilli M, Bartlett CH, Turner NC. Circulating Tumor DNA Markers for Early Progression on Fulvestrant With or Without Palbociclib in ER+ Advanced Breast Cancer. J Natl Cancer Inst . 2021; 113:309-317. doi: 10.1093/jnci/djaa087.

25. Razavi P, Chang MT, Xu G, Bandlamudi C, Ross DS, Vasan N, Cai Y, Bielski CM, Donoghue MTA, Jonsson P, Penson A, Shen R, Pareja F, Kundra R, Middha S, Cheng ML, Zehir A, Kandoth C, Patel R, Huberman K, Smyth LM, Jhaveri K, Modi S, Traina TA, Dang C, Zhang W, Weigelt B, Li BT, Ladanyi M, Hyman DM, Schultz N, Robson ME, Hudis C, Brogi E, Viale A, Norton L, Dickler MN, Berger MF, lacobuzio-Donahue CA, Chandarlapaty S, Scaltriti M, Reis-Filho JS, Solit DB, Taylor BS, Baselga J. The Genomic Landscape of Endocrine-Resistant Advanced Breast Cancers. Cancer Cell. 2018 Sep 10;34(3):427-438.e6. doi: 10.1016/j.ccell.2018.08.008.

26. Van Schooneveld E, Wildiers H, Vergote I, Peter B Vermeulen PB, Dirix LY and Van Laere SJ Dysregulation of microRNAs in breast cancer and their potential role as prognostic and predictive biomarkers in patient management. Breast Cancer Res 2015, 17:21

27. Bertoli G, Cava C, and Castiglioni I. MicroRNAs: New Biomarkers for Diagnosis, Prognosis, Therapy Prediction and Therapeutic Tools for Breast Cancer. Theranostics 2015; 5(10): 1122-1143. doi: $10.7150 /$ thno. 11543

28. Howard EW and Yang X microRNA Regulation in Estrogen Receptor-Positive Breast Cancer and EndocrineTherapy. Biological Procedures Online 2018, 20:17.

29. Zelli V, Compagnoni C, Capelli R, Cannita K, Sidoni T, Ficorella C, Capalbo C, Zazzeroni F, Tessitore A, Alesse E.. Circulating MicroRNAs as Prognostic and Therapeutic Biomarkers in Breast Cancer Molecular Subtypes., J. Pers. Med. 2020; 10, 98; doi:10.3390/jpm10030098

30. Khalife H, Skafi N , Fayyad-Kazan M , Badran B MicroRNAs in breast cancer: New maestros defining the melody. Cancer Genetics 2020; 246-247: 18-40

31. Maillot G, Lacroix-Triki M, Pierredon S, Gratadou L, Schmidt $S$, Bénès $V$, Roché $H$, Dalenc $F$, Auboeuf $D$, Millevoi S, Vagner S. Widespread Estrogen-Dependent Repression of microRNAs Involved in Breast 
Tumor Cell Growth. Cancer Res 2009; 69: 8332-40 DOI: 10.1158/0008-5472.CAN-09-2206.

32. Leivonen S-K, Mäkelä R, Ostling P, Kohonen P, Haapa-Paananen S, Kleivi K, Enerly E, Aakula A, Hellström K, Sahlberg N, Kristensen VN, Børresen-Dale AL, Saviranta P, Perälä M, Kallioniemi O. Protein lysate microarray analysis to identify microRNAs regulating estrogen receptor signaling in breast cancer cell lines. Oncogene. 2009;28:3926-36.

33. Zhao Y, Deng C, Wang J, Xiao J, Gatalica Z, Recker RR, Xiao GG. Let-7 family miRNAs regulate estrogen receptor alpha signaling in estrogen receptor positive breast cancer. Breast Cancer Res Treat 2011 127:69-80.

34. Bailey, S.T, Westerling, T,Brown, M. Loss of estrogen-regulated microRNA expression increases HER2 signaling and is prognostic of poor outcome in luminal breast cancer. Cancer Res. 2015, 75, 436445.

35. Feliciano A, González L, Garcia-Mayea Y, Mir C, Artola M, Barragán N, Martín R, Altés A, Castellvi J, Benavente S, Ramón Y Cajal S, Espinosa-Bravo M, Cortés J, Rubio IT, Leonart ME. Five microRNAs in Serum Are Able to Differentiate Breast Cancer Patients From Healthy Individuals. Front Oncol. 2020 Nov 3;10:586268. doi: 10.3389/fonc.2020.586268

36. Haghi M, Taha MF, Javeri A. Suppressive effect of exogenous miR-16and miR-34a on tumorigenesis of breast cancer cells. J Cell Biochem. 2019;120:13342-13353. https://doi.org/10.1002/jcb.28608

37. Arabkari V, Clancy E, Dwyer RM, Kerin MJ, Kalinina O, Holian E Relative and Absolute Expression Analysis of MicroRNAs Associated with Luminal A Breast Cancer- A Comparison. Pathology \& Oncology Research 2020 26:833-844 https://doi.org/10.1007/s12253-019-00627-y

38. Zhou Q, Zeng H,Ye P, Shi Y, Guo J and Long X Differential microRNA profiles between fulvestrantresistant and tamoxifen-resistant human breast cancer cells Anti-Cancer Drugs 2018, 29:539-548.

39. Guo J, He K, Zeng H, Shi Y, Ye P, Zhou Q, Pan Z, Long X. Differential microRNA expression profiles determined by next generation sequencing in three fulvestrant resistant human breast cancer cell lines. Oncol Letters 2019; 17: 3765-3776.

40. Rao X., Di Leva G., Li M., Fang F., Devlin C., Hartman-Frey C., Burow M.E., Ivan M., Croce C.M. , Nephew K.P. MicroRNA-221/222 confers breast cancer fulvestrant resistance by regulating multiple signaling pathways. Oncogene 2011, 30, 1082-1097.

41. Yu X., Li R., Shi W., Jiang T., Wang Y., Li C., Qu X. Silencing of microRNA-21 confers the sensitivity to tamoxifen and fulvestrant by enhancing autophagic cell death through inhibition of the PI3K-AKTmTOR pathway in breast cancer cells. Biomed. Pharmacother. 2016, 77, 37-44.

\section{Tables}

Table 1. Patient and tumor characteristics 


\begin{tabular}{|c|c|c|}
\hline & $\mathbf{N}$ & $\%$ \\
\hline Age in years, median (range) & 67 & $(32 ; 92)$ \\
\hline \multicolumn{3}{|l|}{ Menopausal status, n (\%) } \\
\hline Pre/peri-menopausal & 15 & 25.4 \\
\hline Post-menopausal & 43 & 72.9 \\
\hline Unknown & 1 & 1.7 \\
\hline \multicolumn{3}{|l|}{ HR status, n (\%) } \\
\hline $\mathrm{ER}+/ \mathrm{PgR}+$ & 34 & 57.6 \\
\hline $\mathrm{ER}+/ \mathrm{PgR}-$ & 24 & 40.7 \\
\hline Unknown & 1 & 1.7 \\
\hline \multicolumn{3}{|l|}{ HER2 status, n (\%) } \\
\hline Negative & 51 & 86.4 \\
\hline Positive & 5 & 8.5 \\
\hline Unknown & 3 & 5.1 \\
\hline \multicolumn{3}{|l|}{ Ki67 } \\
\hline$\leq 20$ & 24 & 40.7 \\
\hline$>20$ & 24 & 40.7 \\
\hline Unknown & 1 & 1.7 \\
\hline \multicolumn{3}{|l|}{ Tumor subtype } \\
\hline Luminal A & 24 & 40.7 \\
\hline Luminal B & 27 & 45.8 \\
\hline Luminal HER2 & 3 & 5.1 \\
\hline Unknown & 5 & 8.5 \\
\hline \multicolumn{3}{|l|}{ Metastatic sites (number) } \\
\hline$\leq 3$ & 54 & 91.5 \\
\hline$>3$ & 5 & 8.5 \\
\hline \multicolumn{3}{|l|}{ Metastatic sites } \\
\hline Bone-only & 9 & 15.3 \\
\hline Visceral & 28 & 47.5 \\
\hline
\end{tabular}




\section{Previous therapy with NSAI}

\begin{tabular}{lcc|}
\hline Metastatic setting & 12 & 20.3 \\
\hline Adjuvant setting & 19 & 32.2 \\
\hline Both & 1 & 1.7 \\
\hline Missing & 27 & 45.8 \\
\hline
\end{tabular}

Prior lines of therapy for metastatic disease

\begin{tabular}{lll}
\hline 0 & 8 & 13.6 \\
\hline 1 & 10 & 16.9 \\
\hline 2 & 13 & 22.0 \\
\hline$\geq 3$ & 27 & 45.8 \\
\hline Unknown & 1 & 1.7 \\
\hline Status at Fulvestrant & & \\
\hline Progressive disease & 48 & 81.4 \\
\hline Response or stable disease & 11 & 18.6
\end{tabular}

Table 2: miRNAs associated with outcome 


\begin{tabular}{|c|c|c|c|c|c|c|c|c|c|}
\hline \multirow{3}{*}{ mIRNA } & \multicolumn{5}{|c|}{ PFS } & \multicolumn{4}{|c|}{ 18-month PFS } \\
\hline & $\mathrm{N}$ & HR & $95 \% \mathrm{Cl}$ & & & & & & \\
\hline & & & & & $\begin{array}{l}\mathrm{p} \\
\text { value }\end{array}$ & OR & $95 \% \mathrm{Cl}$ & & $\begin{array}{l}p \\
\text { value }\end{array}$ \\
\hline hsa-miR-549a & 16 & 1,06 & 1,016 & 1,105 & 0,007 & 0,917 & 0,844 & 0,996 & 0,041 \\
\hline hsa-miR-644a & 16 & 1,034 & 1,004 & 1,064 & 0,027 & 0,928 & 0,863 & 0,999 & 0,047 \\
\hline hsa-let-7c-5p & 48 & 0,994 & 0,988 & 0,999 & 0,022 & 1,016 & 1,004 & 1,029 & 0,012 \\
\hline hsa-miR-16-5p & 37 & 1,008 & 0,999 & 1,017 & 0,071 & 0,939 & 0,89 & 0,991 & 0,022 \\
\hline hsa-miR-4536-5p & 15 & 1,035 & 1,002 & 1,069 & 0,038 & & & & \\
\hline hsa-miR-451a & 19 & 1,006 & 1,001 & 1,011 & 0,015 & & & & \\
\hline hsa-miR-585-3p & 12 & 1,191 & 1,026 & 1,382 & 0,021 & & & & \\
\hline hsa-miR-548a-5p & 10 & 1,307 & 1,002 & 1,706 & 0,049 & & & & \\
\hline hsa-miR-548g-3p & 14 & 1,093 & 1,022 & 1,169 & 0,009 & & & & \\
\hline hsa-miR-1285-5p & 11 & 1,052 & 1 & 1,107 & 0,049 & & & & \\
\hline hsa-miR-1290 & 16 & 1,029 & 1,003 & 1,055 & 0,026 & & & & \\
\hline hsa-miR-888-5p & 16 & 1,117 & 1,018 & 1,226 & 0,020 & & & & \\
\hline hsa-miR-200b-3p & 21 & 1,043 & 1,006 & 1,082 & 0,024 & & & & \\
\hline hsa-miR-1297 & 16 & 1,035 & 1,004 & 1,066 & 0,024 & & & & \\
\hline $\begin{array}{l}\text { hsa-miR-548z+hsa- } \\
\text { miR-548h-3p }\end{array}$ & 16 & 1,028 & 1,001 & 1,055 & 0,038 & & & & \\
\hline hsa-let-7f-5p & 11 & 0,798 & 0,671 & 0,948 & 0,011 & & & & \\
\hline
\end{tabular}

PFS: progression-free survival; HR Hazard ratio; $95 \% \mathrm{Cl} 95 \%$ confidence interval; OR Odd Ratio In bold miRNA which are statistically significantly associated with 18-month PFS $(p<.05)$

\section{Supplementary Files}

This is a list of supplementary files associated with this preprint. Click to download.

- supplementarytable1.doc

- supplementarytable2.xlsx 